\title{
A Marker-Based Approach for the Automated Selection of a Single Segmentation From a Hierarchical Set of Image Segmentations
}

\author{
Yuliya Tarabalka, Member, IEEE, James C. Tilton, Senior Member, IEEE, Jón Atli Benediktsson, Fellow, IEEE, \\ and Jocelyn Chanussot, Senior Member, IEEE
}

\begin{abstract}
The Hierarchical SEGmentation (HSEG) algorithm, which combines region object finding with region object clustering, has given good performances for multi- and hyperspectral image analysis. This technique produces at its output a hierarchical set of image segmentations. The automated selection of a single segmentation level is often necessary. We propose and investigate the use of automatically selected markers for this purpose. In this paper, a novel Marker-based HSEG (M-HSEG) method for spectral-spatial classification of hyperspectral images is proposed. Two classification-based approaches for automatic marker selection are adapted and compared for this purpose. Then, a novel constrained marker-based HSEG algorithm is applied, resulting in a spectral-spatial classification map. Three different implementations of the M-HSEG method are proposed and their performances in terms of classification accuracies are compared. The experimental results, presented for three hyperspectral airborne images, demonstrate that the proposed approach yields accurate segmentation and classification maps, and thus is attractive for remote sensing image analysis.
\end{abstract}

Index Terms-Classification, hierarchical segmentation, hyperspectral images, marker selection.

\section{INTRODUCTION}

$\mathbf{O}$ NE OF THE MOST challenging problems currently addressed by the remote sensing community is the development of effective analysis and processing techniques for data acquired with the last generation of imaging sensors [1]. In particular, hyperspectral imaging is a relatively new technique that records the energy of the received light in tens or hundreds of narrow spectral bands in each spatial position in the image [2].

Manuscript received March 22, 2011; revised June 29, 2011; accepted October 17, 2011. Date of publication November 15, 2011; date of current version February 29,2012. This research was supported by an appointment to the NASA Postdoctoral Program at the Goddard Space Flight Center, administered by Oak Ridge Associated Universities through a contract with NASA.

Y. Tarabalka is with the NASA Goddard Space Flight Center, Greenbelt, MD 20771 USA (corresponding author, e-mail: yuliya.tarabalka@nasa.gov).

J. C. Tilton is with the NASA Goddard Space Flight Center, Greenbelt, MD 20771 USA (e-mail: james.c.tilton@nasa.gov).

J. A. Benediktsson is with the Faculty of Electrical and Computer Engineering, University of Iceland, 107 Reykjavik, Iceland (e-mail: benedikt@hi.is)

J. Chanussot is with the Grenoble Images Speech Signals and Automatics Laboratory (GIPSA-Lab), Grenoble Institute of Technology (INPG), 38402 Saint-Martin-d'Hères Cedex, France (e-mail: jocelyn.chanussot@gipsa-lab. grenoble-inp.fr).

Color versions of one or more of the figures in this paper are available online at http://ieeexplore.ieee.org.

Digital Object Identifier 10.1109/JSTARS.2011.2173466
Therefore, it becomes possible to identify physical materials and classify regions within the image scene with much higher accuracies when compared to panchromatic or multispectral sensors. Remote sensing image classification, which can be defined as identification of objects in a scene captured by a remote imaging sensor, is an important task in many application domains such as precision agriculture, monitoring of environment, urban planning, etc.

Most of the previously proposed classification methods process each pixel independently using its spectral values only [3]-[6]. One of the most frequently used techniques is Support Vector Machines (SVM) [7], [8]. Recent studies have shown the advantage of including information about spatial dependencies for accurate image analysis, i.e., performing spectral-spatial classification [9]-[12].

In the seminal works on spectral-spatial image classification, the information from the closest neighborhoods, defined by either fixed windows [9], [13] or morphological profiles [14], has been considered for classifying each pixel. In our recent works, we have shown the advantage of using segmentation for distinguishing spatial structures in a hyperspectral image [15], [16]. Segmentation can be defined as an exhaustive partitioning of the input image into homogeneous regions (with respect to some criterion of interest, e.g., intensity or texture) [17], [18]. Different techniques have been investigated for segmentation of hyperspectral data, such as watershed, partitional clustering, and Hierarchical SEGmentation (HSEG). Then, each region from a segmentation map was classified by applying a majority vote rule over the pixelwise SVM classification results [16].

The HSEG method is one of the few available segmentation approaches in the state-of-art that naturally integrates spatial and spectral information. HSEG is a combination of region object finding by hierarchical step-wise optimization (HSWO, or iterative best merge region growing) [19] and region clustering by grouping spectrally similar but spatially disjoint regions [20], [21]. Unlike most other segmentation approaches, the HSEG produces at its output a segmentation hierarchy. A segmentation hierarchy is defined as a set of image segmentations at different levels of detail, in which segmentations at coarser levels of details are produced by merging regions at finer levels of detail. In this hierarchy, an object of interest may be represented by several regions at finer levels of detail, and may be merged with another region at coarser levels of detail. It is often necessary to choose a single optimum hierarchical segmentation level, which 
depends on the specific application (e. g., the specific classification problem). For instance, when performing urban data analysis, a coarser segmentation map may divide an image into impervious/non-impervious surfaces, while a finer segmentation map may be composed of regions representing individual buildings, trees, etc. In [16], we have selected an appropriate level of segmentation detail for spectral-spatial classification ${ }^{1}$ interactively with the program HSEGViewer [21]. However, an automatic procedure would be desirable. Plaza and Tilton [22] proposed to use joint spectral/spatial homogeneity scores computed from the segmented regions, for automating the selection of relevant hierarchical level(s). This approach may be computationally expensive for large data sets.

The main focus of this paper is to propose a method for the automated selection of a single hierarchical segmentation level. This objective can be achieved by incorporation of some additional knowledge into a segmentation procedure. We propose and investigate the use of automatically derived markers, or region seeds, for this purpose. A marker in our study is defined as a set of image pixels (which are not necessarily spatially connected) associated with one object in the image scene. The marker-controlled segmentation approach determines a marker for each region of interest and then segments an image in such a way that each region in a segmentation map contains one marker. In order to accurately segment an image, a marker for each image object/region must be selected.

The problem of automatic marker selection has been discussed in previous studies. Markers are often chosen by searching flat zones (i.e., connected components of pixels of constant gray-level value), zones of homogeneous texture, or image extrema [23]. Gómez et al. [24] used histogram analysis to obtain a set of representative pixel values, and the markers were generated with all the image pixels having representative gray values. Noyel et al. [25], [26] performed classification of the hyperspectral image (using different methods, such as Clara [27] and linear discriminant analysis [28]) and then filtered the classification maps class by class, using mathematical morphology operators, for selecting large spatial regions as markers. In [12], we have proposed to use probability estimates obtained by the pixelwise SVM classification in order to choose the most reliably classified pixels as markers of spatial regions. Furthermore, a Minimum Spanning Forest (MSF) rooted on the selected markers was built, resulting in a spectral-spatial classification map. One of the advantages of applying classification-based methods for marker selection is that segmentation regions grown from these markers can be immediately assigned to the class of the corresponding classification-derived marker, thus yielding a classification map.

In this paper we propose a new Marker-based HSEG (M-HSEG) method for segmentation and spectral-spatial classification of hyperspectral images. Two classification-based approaches for marker selection proposed in [12] and [26] are adapted and compared in order to define relevant markers for the HSEG procedure. First, markers of spatial regions are au-

\footnotetext{
${ }^{1}$ In [16], we verified that the interactively selected hierarchical level was optimal by quantitatively evaluating the segmentation results at several hierarchical levels versus the test data and retaining the best results.
}

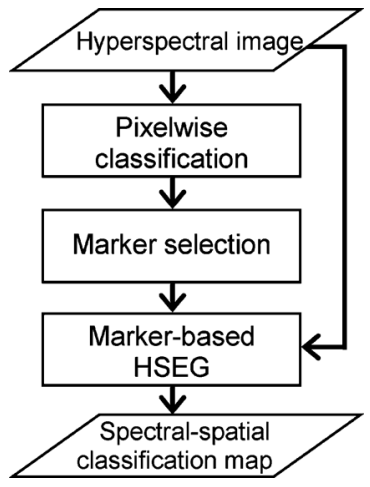

Fig. 1. Flow-chart of the proposed $M-H S E G$ classification scheme.

tomatically selected using classification results. Then, a novel constrained M-HSEG algorithm is applied, resulting in both segmentation and classification maps. We propose and discuss several ways of integrating markers into the HSEG algorithm.

Although the proposed marker-based segmentation scheme was designed for hyperspectral data, the method is general and can be applied to other types of data as well. Experimental results are demonstrated on three hyperspectral airborne images acquired by the Reflective Optics System Imaging Spectrometer (ROSIS), the Airborne Visible/Infrared Imaging Spectrometer (AVIRIS), and the Airborne Imaging System for different Applications (AISA) Eagle Sensor. The proposed M-HSEG approach is compared with the recently proposed marker-based classification technique, which consists in constructing an MSF from a set of markers [12], as well as with several other spectral-spatial classification techniques.

The outline of this paper is as follows. The next section describes two classification-based approaches for marker selection adapted for the proposed M-HSEG scheme. In Section III, the proposed constrained M-HSEG method and its implementations are presented. Section IV presents an MSF algorithm used for comparison. Experimental results are discussed in Section $\mathrm{V}$ and conclusions are drawn in Section VI.

\section{Classification-Based Marker Selection}

The flow-chart of the proposed M-HSEG classification method is depicted in Fig. 1. An input $B$-band hyperspectral image can be considered as a set of $n$ pixel vectors $\mathbf{X}=\left\{\mathbf{x}_{j} \in \mathbb{R}^{B}, j=1,2, \ldots, n\right\}$. Let $\Omega=\left\{\omega_{1}, \omega_{2}, \ldots, \omega_{K}\right\}$ be a set of information classes in the image scene. Classification consists in assigning each pixel to one of the $K$ classes of interest.

Because in our study markers are automatically selected using classification results, the preliminary step for both marker selection techniques consists in performing a pixelwise classification. In the following, two marker selection techniques adapted for the M-HSEG method are described.

\section{A. Morpho-MS Approach}

The Morpho-MS marker selection technique follows the approach proposed in [26], which is based on morphological filtering of the classification map. The proposed method is based on an assumption that if a pixelwise classifier has assigned a 
large set of spatially connected pixels to the same class, the corresponding region is relevant and must contain a marker. The Morpho-MS technique consists of the following steps:

1) Perform a pixelwise classification of a hyperspectral image. We propose to use an SVM classifier for this purpose, which is very well suited to classify high-dimensional data when a limited number of training samples is available [7], [29]. We refer the reader to [7], [30] for details on the SVM technique. This step results in a classification map $\mathbf{L}=\left\{L_{j}, j=1,2, \ldots, n\right\}$, where each pixel $\mathbf{x}_{j}$ has a class label $L_{j}$.

2) Generate $K$ binary images $h_{\omega_{k}}(k=1, \ldots, K)$ associated with $K$ classes, so that pixels assigned to the class $\omega_{k}$ have the value $k$ in the image for the corresponding class:

$$
h_{\omega_{k}}(j)= \begin{cases}k, & \text { if } L_{j}=k \\ 0, & \text { otherwise }\end{cases}
$$

3) Perform morphological erosion of each image $h_{\omega_{k}}$ by a preset structuring element (SE) $N$ corresponding to the pixel neighborhood. The erosion assigns a zero value to the pixels situated on the boundaries of objects in the image scene (assuming that the uncertainty of correct classification on the boundaries is higher than in the center of objects). The remaining non-zero pixels correspond to the cores (centers) of large spatial structures, which are assumed to be reliably classified and therefore can be used as region markers. The choice of an SE depends on the size of objects of interest in the image. We propose to use an elementary $3 \times 3$ square $\mathrm{SE}$, in order to minimize the risk of loosing small spatial structures when selecting markers.

4) Combine $K$ images $h_{\omega_{k}}^{\prime}$ using the pixelwise maximum operator:

$$
\mathbf{L}^{\prime}(j)=\max _{k=1, \ldots, K}\left(h_{\omega_{k}}^{\prime}(j)\right)
$$

5) Perform a connected component labeling of $\mathbf{L}^{\prime}$, using an eight-neighborhood connectivity [31].

6) Finally, select each connected component with non-zero values $(k)$ in $\mathbf{L}^{\prime}$ as a marker, with the corresponding class label $(k)$.

The resulting marker map contains markers (spatially connected sets of pixels) for large, i.e., reliably classified regions. The drawback of the Morpho-MS technique is that it is not able to define a marker for a spatial object which is smaller than the SE. Therefore, small objects in the image risk to be assimilated with their neighboring structures.

\section{B. Proba-MS Approach}

A second marker selection technique, Proba-MS (proposed in [12]), mitigates the drawback of the Morpho-MS approach, by analyzing both the sizes of connected components in the classification map and the probability estimates of correct classification. The Proba-MS method consists of two steps:

1) Probabilistic Pixelwise Classification: Perform a probabilistic pixelwise SVM classification of a hyperspectral image [8], [30]. This step results in a classification map (where each pixel has a unique class label) and a probability map (containing probability estimates for each pixel to belong to the assigned class).

Standard SVM classifications do not provide probability estimates for the individual classes. In order to get these estimates, pairwise coupling of binary probability estimates can be applied [8], [32]. In our research we use the probabilistic SVM method implemented in LIBSVM library [8]. The objective is to estimate, for each pixel $\mathbf{x}$, classification probabilities:

$$
\mathbf{p}=\left\{p_{k}=p\left(L_{\mathbf{x}}=k \mid \mathbf{x}\right), k=1, \ldots, K\right\}
$$

For this purpose, first pairwise class probabilities $r_{i j} \approx p\left(L_{\mathbf{x}}=i \mid L_{\mathbf{x}}=i\right.$ or $\left.j, \mathbf{x}\right)$ are estimated, and then the probabilities in (3) are computed, as described in [32]. Finally, a probability map is constructed, by assigning to each pixel the maximum probability estimate $\max \left(p_{k}\right), k=1, \ldots, K$.

2) Marker Selection: Apply a connected component labeling on the classification map, using an eight-neighborhood connectivity [31]. Then, analyze each connected component as follows:

- if a region is large (number of pixels in the region > $M)$, it is considered to be relevant, and its marker is defined as the $P \%$ of pixels within this region with the highest probability estimates.

- If a region is small, it is further investigated if its pixels were classified to a particular class with a high probability. Its potential marker is formed by the pixels with probability estimates higher than a defined threshold $S$.

The procedure of the choice of parameters $(M, P, S)$ for the Proba-MS technique is described in [12]. At the output of the marker selection step, a map of $m$ markers is obtained, where each marker $\mathbf{O}_{i}=\left\{\mathbf{x}_{j} \in \mathbf{X}, j=\right.$ $\left.1,2, \ldots, \operatorname{card}\left(\mathbf{O}_{i}\right) ; L_{\mathbf{O}_{i}}\right\}(i=1, \ldots, m)$ consists of one or several pixels $\left(\operatorname{card}\left(\mathbf{O}_{i}\right)=1\right.$ or $\operatorname{card}\left(\mathbf{O}_{i}\right)>1$, respectively) and has a class label $L_{\mathbf{O}_{i}}$. One should note that a marker is not necessarily a spatially connected set of pixels.

\section{MARKER-BASED HSEG}

A key feature of the HSEG algorithm is the tight intertwining of region growing segmentation (using the HSWO approach [19]), which produces spatially connected regions, with region clustering, which groups together similar spatially disjoint regions [20], [21]. The following outline of HSEG summarizes the description given in [21]:

1) Initialization: Initialize the segmentation by assigning each image pixel a region label. If a pre-segmentation is provided, label each pixel according to the presegmentation. Otherwise, label each pixel as a separate region.

1) Calculate the Dissimilarity Criterion (DC) value between all pairs of spatially adjacent regions. A spatially adjacent region for a given region is the one containing pixels situated in the neighborhood (eight-neighborhood is used in our study) of the given region's pixels.

2) Find the smallest DC value dissim_val and set thresh_val equal to it. Then, merge all pairs of spatially adjacent regions with dissim_val $=$ thresh_val.

3) If the parameter $S_{w g h t}>0.0$, merge all pairs of spatially non-adjacent regions with dissim_val $\leq S_{w g h t}$. thresh_val. 


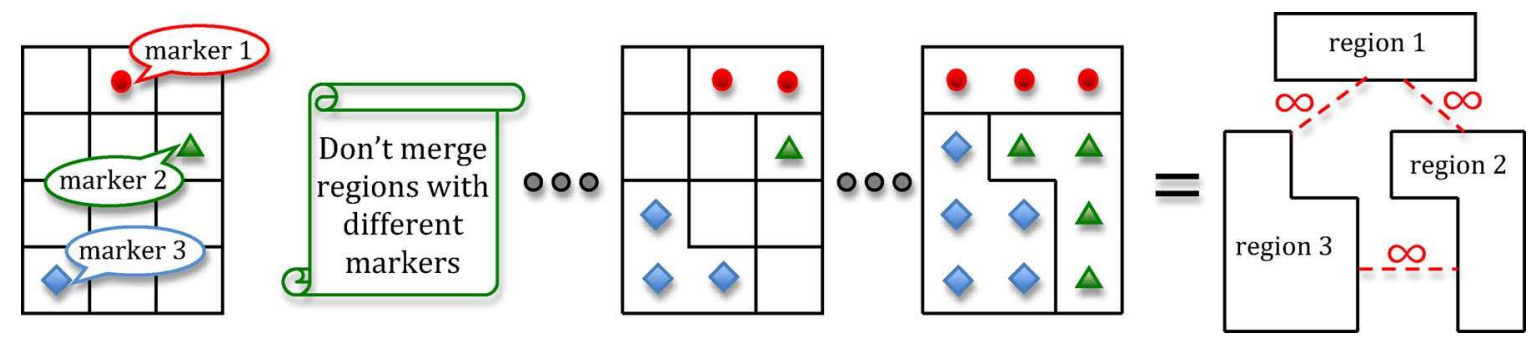

Fig. 2. Scheme illustrating the $M-H S E G$ algorithm.

4) Stop if no more merges are required (convergence is achieved). Otherwise, return to step 1.

Different measures can be applied for computing DCs between regions, such as vector norms and Spectral Angle Mapper (SAM) between the region mean vectors [21]. The $L 1$ vector norm between two vectors $\mathbf{u}_{i}=\left(u_{i 1}, \ldots, u_{i B}\right)^{T}$ and $\mathbf{u}_{j}=$ $\left(u_{j 1}, \ldots, u_{j B}\right)^{T}$ is defined as

$$
L 1\left(\mathbf{u}_{i}, \mathbf{u}_{j}\right)=\sum_{b=1}^{B}\left|u_{i b}-u_{j b}\right| .
$$

The vector Infinity (Inf) norm ( $\infty$-norm) between $\mathbf{u}_{i}$ and $\mathbf{u}_{j}$ is given as

$$
\operatorname{Inf}\left(\mathbf{u}_{i}, \mathbf{u}_{j}\right)=\left\|\mathbf{u}_{i}-\mathbf{u}_{j}\right\|_{\infty}=\max _{b=1, \ldots, B}\left|u_{i b}-u_{j b}\right|
$$

The SAM measure between $\mathbf{u}_{i}$ and $\mathbf{u}_{j}$ determines the spectral similarity between two vectors by computing the angle between them. It is computed as

$$
S A M\left(\mathbf{u}_{i}, \mathbf{u}_{j}\right)=\arccos \left(\frac{\sum_{b=1}^{B} u_{i b} u_{j b}}{\left[\sum_{b=1}^{B} u_{i b}^{2}\right]^{1 / 2}\left[\sum_{b=1}^{B} u_{j b}^{2}\right]^{1 / 2}}\right) .
$$

The optional parameter $S_{w g h t}$, in HSEG, tunes the relative importance of clustering based on spectral information only versus region growing. If $S_{w g h t}=0.0$, only spatially adjacent regions are allowed to merge. If $0.0<S_{w g h t} \leq 1.0$, spatially adjacent merges are favored compared with spatially nonadjacent merges by a factor of $1.0 / S_{w g h t}$.

The allowance for the merging of spatially non-adjacent regions in HSEG leads to heavy computational demands. In order to reduce these demands, a recursive divide-and conquer approximation of HSEG (RHSEG) has been developed. The NASA Goddard RHSEG software provides an efficient parallel implementation of the RHSEG algorithm.

HSEG naturally produces a segmentation hierarchy consisting of a set of segmentations from initialization down to the final trivial one region segmentation (if allowed to proceed that far). However, for practical applications a subset of one or several segmentations needs to be selected out from this hierarchy. The proposed M-HSEG algorithm is designed for automatically selecting the segmentation level of detail for the most accurate segmentation and classification result.

\section{A. M-HSEG Algorithm}

The main idea behind the marker-based HSEG algorithm consists in assigning a marker label for each region containing marker pixels, and merging regions with an additional condition: two regions with different marker labels can not be merged together (see Fig. 2). The proposed M-HSEG algorithm can be summarized as follows:

1) Initialize the segmentation by labeling either the whole marker (i.e., all the pixels belonging to the same marker), or an individual non-marker pixel as one region. Assign for every region the corresponding marker label (which is equal to zero for non-marked regions).

2) At each iteration, perform HSEG, with an additional condition: two regions with different non-zero marker labels have the DC equal to infinity (in practice, the upper maximum value of float) and are never merged together. When a marked region is merged with a non-marked region, the resulting region keeps the marker label inherited from the marked region.

3) Stop the iterative process when either no more merging is possible (number of regions is equal to the number of markers) or the smallest DC between any two neighboring regions is higher than the preset (or computed) threshold.

4) Classification: Assign the class of each marker to all pixels in the region containing this marker. If the segmentation result contains non-marked regions (i.e., M-HSEG converged before the number of regions was equal to the number of markers), classify these regions. For instance, this can be done by applying a majority vote rule over the pixelwise classification results [16]: for every non-marked region, all the pixels are assigned to the most frequent class within this region.

\section{B. Implementations of the M-HSEG Algorithm}

We have investigated the performance of three different implementations of the proposed M-HSEG method. One implementation, $\mathrm{M}_{-} \mathrm{HSEG}^{r}$, is based on the description given in the previous subsection. All the pixels belonging to the same marker are initialized as one region, and then iterative region merging is performed.

A second implementation, $\mathrm{M}-\mathrm{HSEG}^{p}$, first initializes each pixel as one region and assigns a marker label for every region (equal to zero for non-marked regions). Thus, after the initialization, multiple regions can have the same non-zero marker label. During the region merging procedure, the regions with equal non-zero marker labels have a zero DC value, while the 


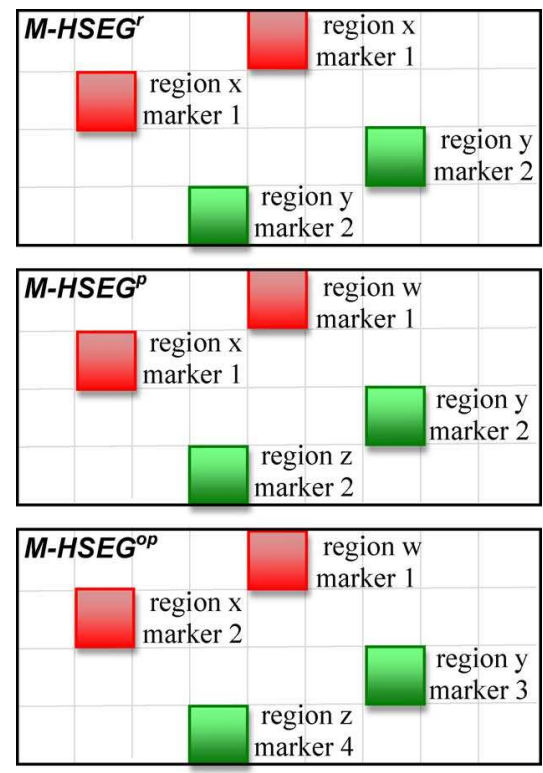

Fig. 3. Scheme illustrating region and marker labeling of marker pixels at the initialization step of three different implementations of the M-HSEG algorithm: initial markers 1 and 2 are denoted by red and green colors, respectively.

regions with different non-zero marker labels have the DC equal to infinity.

A third implementation, M-HSEG ${ }^{o p}$, first initializes each pixel as one region. If the given pixel is marked, the corresponding region obtains a new non-zero marker label, with the corresponding information class. Thus, at the initialization step all the markers are split into one-pixel markers. Then, iterative region merging is performed, providing that regions with different markers cannot be merged together. At the final step, the regions containing pixels of the same initial marker are merged together.

Fig. 3 illustrates a schematic example of region and marker labeling of marker pixels at the initialization step of the three proposed implementations of M-HSEG. The implementations

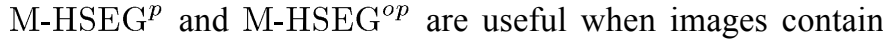
large regions with high intra-region spectral variation. In this case, it may be advantageous to compute region feature vectors over parts of these regions.

\section{CONSTRUCTION OF AN MSF}

This section recapitulates the marker-based classification technique recently proposed in [12] for hyperspectral image analysis, used for comparison with the novel M-HSEG method. It consists in constructing an MSF rooted on a set of markers.

Each image pixel is considered as a vertex $v \in V$ of an undirected graph $G=(V, E, W)$, where $V$ and $E$ are the sets of vertices and edges, respectively, and $W$ is a weighting function. Each edge $e_{i, j} \in E$ of this graph connects a couple of vertices $i$ and $j$ corresponding to the neighboring pixels (we assume eight-neighborhood in our study). A weight $w_{i, j}$ is assigned to each edge $e_{i, j}$, which indicates the degree of dissimilarity between two vertices (i.e., two corresponding pixels) connected by this edge. Different dissimilarity measures can be used for

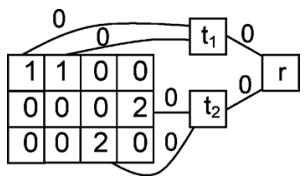

Fig. 4. Example of addition of extra vertices $t_{1}, t_{2}, r$ to the image graph for construction of the MSF rooted on markers 1 and 2; non-marker pixels are denoted by " 0 ",

computing weights of edges, such as vector norms and SAM between two pixel vectors.

Given a graph $G=(V, E, W)$, a spanning forest $F=\left(V, E_{F}\right)$ of $G$ is a non-connected graph without cycles such that $E_{F} \subset E$. The MSF rooted on a set of $m$ distinct vertices $\left\{t_{1}, \ldots, t_{m}\right\}$ is defined as a spanning forest $F^{*}=\left(V, E_{F^{*}}\right)$ of $G$, such that each tree of $F^{*}$ is grown from one root $t_{i}$, and the sum of the edges weights of $F^{*}$ is minimal [33]:

$$
F^{*} \in \arg \min _{F \in S F}\left\{\sum_{e_{i, j} \in E_{F}} w_{i, j}\right\},
$$

where $S F$ is a set of all spanning forests of $G$ rooted on $\left\{t_{1}, \ldots, t_{m}\right\}$.

In order to obtain the MSF rooted on markers, $m$ additional vertices $t_{i}, i=1, \ldots, m$, are introduced. Each extra vertex $t_{i}$ is connected by the edge with a null weight to the pixels representing a marker $\mathbf{O}_{i}$. Furthermore, an additional root vertex $r$ is added and is connected by the null-weight edges to the vertices $t_{i}$ (see an example on Fig. 4). The minimum spanning tree [33] of the built graph induces an MSF in $G$, where each tree is grown on a vertex $t_{i}$. The MSF is obtained after removing the vertex $r$. Prim's algorithm can be used for building the MSF (we refer the readers to [12], [34] for details on this algorithm).

Each tree in the MSF corresponds to a region in the segmentation map. A classification map is obtained by assigning the class of each marker to all the pixels grown from this marker.

\section{EXPERIMENTAL RESULTS}

Three hyperspectral data sets were used for the experiments, with different contexts (one urban area and two agricultural areas) and acquired by different sensors (ROSIS, AVIRIS and AISA Eagle) with different spectral and spatial resolutions. These data sets and the corresponding results are presented in the next three sections.

\section{A. Classification of Center of Pavia Image}

The Center of Pavia image was acquired by the ROSIS optical sensor over the urban area of Pavia, Italy. The image has spatial dimensions of 785 by 300 pixels, with a spatial resolution of $1.3 \mathrm{~m} /$ pixel and 102 spectral channels. Nine classes of interest are considered, which are detailed in Table I, with the number of labeled samples for each class. Fig. 5 shows a three-band false color image and the reference data. Thirty samples for each class were randomly chosen from the reference data as training samples. The remaining samples composed the test set. The training set was used for training an SVM classifier, while the test set was 


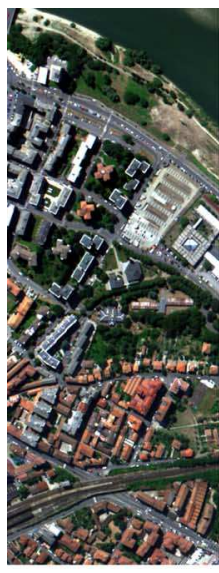

(a)

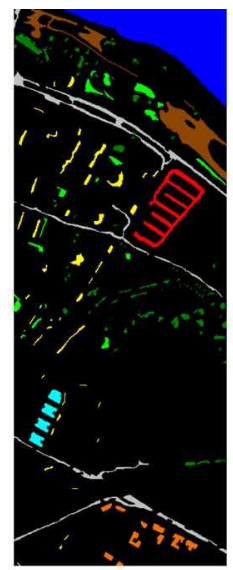

(b)

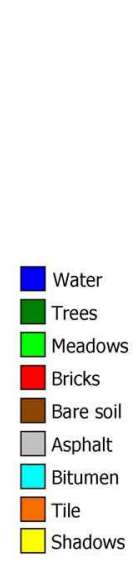

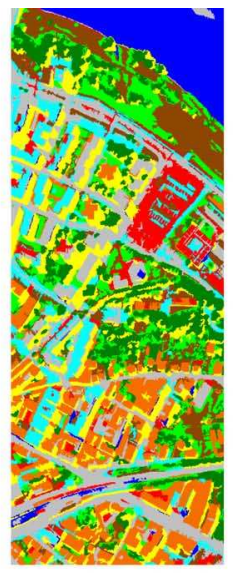

(c)

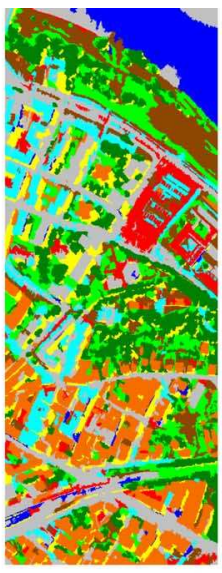

(d)

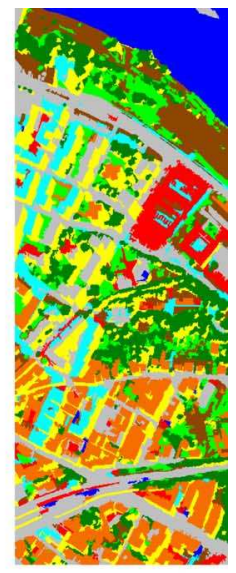

(e)

Fig. 5. Center of Pavia image. (a) Three-band color composite. (b) Reference data. (c) M-HSEG ${ }^{o p}$ classification map (Proba-MS marker selection, SAM DC, $S_{w g h t}=0.2$ ). (d) MSF classification map (Proba-MS marker selection, SAM DC). (e) M-HSEG ${ }^{o p}$ classification map (Morpho-MS marker selection, SAM DC, $\left.S_{w g h t}=0.0\right)$.

TABLE I

Results for the Center of Pavia Data Set. Information Classes, Number of Labeled Samples and Classification Accuracies in Percentage, Using the Proba-MS Marker Selection Approach: Overall Accuracy (OA), Average Accuracy (AA), Kappa Coefficient ( $\kappa$ ) and Class-SpeCific ACCuracies. The Highest ACCuracies are Bolded IN EACH CATEGORY

\begin{tabular}{|c|c|c|c|c|c|c|c|c|c|c|c|c|}
\hline \multirow{3}{*}{$\frac{\overline{\mathrm{DC}}}{S_{w g h t}}$} & \multirow{3}{*}{$\begin{array}{l}\text { Number } \\
\text { of labeled } \\
\text { samples }\end{array}$} & \multicolumn{2}{|c|}{ M-HSEG $^{r}$} & \multicolumn{2}{|c|}{ M-HSEG $^{p}$} & \multicolumn{4}{|c|}{ M-HSEG ${ }^{o p}$} & \multirow{3}{*}{ SVM } & \multicolumn{2}{|c|}{ MSF } \\
\hline & & L1 & SAM & L1 & SAM & L1 & SAM & SAM & SAM & & L1 & SAM \\
\hline & & \multicolumn{6}{|c|}{0.0} & 0.2 & 0.5 & & & \\
\hline $\mathrm{OA}$ & & 90.44 & 91.32 & 92.44 & 92.86 & 95.96 & 96.20 & 96.35 & 95.50 & 94.96 & 96.74 & 91.3 \\
\hline & & 85.69 & 88.03 & 9.59 & 90.43 & 5.00 & 5.26 & 5.49 & 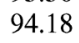 & 56 & 5.66 & 92.6 \\
\hline & & 87.90 & 89.02 & 90.43 & 90.96 & 4.88 & 95.18 & 95.37 & 94.30 & 3.61 & 5.86 & 39.1 \\
\hline Water & 12734 & 97.41 & 97.80 & 97.41 & 97.80 & 97.41 & 97.74 & 97.74 & 97.69 & 8.12 & 98.63 & 37.9 \\
\hline Tree & 2405 & 83.75 & 71.03 & 63 & 78.44 & 40 & 90.32 & 90.36 & 77 & & 2.51 & 39.1 \\
\hline & 1788 & 93.34 & 91.70 & 94.08 & 89.31 & 20 & 95.45 & 95.62 & 69 & & 5.51 & 3.52 \\
\hline & & 8 & 7 & 57 & 75.36 & 27 & 85.97 & 87.77 & 02 & & 5.59 & 87.8 \\
\hline & 46 & 87.39 & 92.47 & 86.66 & 92.45 & 99.29 & 99.31 & 99.33 & 9.01 & 7.12 & 99.29 & 98.0 \\
\hline & 4844 & 88.55 & 89.26 & 92.33 & 92.85 & 93.77 & 94.91 & 95.01 & 92.48 & 3.52 & 95.10 & 93.0 \\
\hline Bitumen & 972 & 78.98 & 86.62 & 84.82 & 88.96 & 93.63 & 94.80 & 94.80 & 92.46 & 2.48 & 94.37 & 92.57 \\
\hline Tile & 1112 & 71.44 & 93.81 & 82.72 & 98.89 & 98.06 & 98.80 & 98.80 & 96.49 & 97.41 & 99.91 & 100 \\
\hline Shadows & 2020 & 100 & 98.64 & 100 & 99.80 & 100 & 100 & 100 & 100 & 99.95 & 100 & 91.6 \\
\hline
\end{tabular}

employed for estimating classification accuracies of the considered algorithms.

The multiclass one-versus-one SVM classification, with the Gaussian radial basis function (RBF) kernel, of the hyperspectral image was performed. The optimal parameters $C$ (penalty during the SVM optimization) and $\gamma$ (spread of the RBF kernel) were chosen by fivefold cross validation: $C=128, \gamma=2^{-5}$.

Then, marker selection was performed, using Proba-MS and Morpho-MS approaches. The Proba-MS method was applied with parameters $M=20, P=40 \%$. The threshold $S$ was chosen to be equal to the lowest probability within the highest $2 \%$ of the probability estimates for the whole image. These parameters were chosen (and applied for all three data sets) following the recommendations in [12], where it was shown that the method was robust to the selection of parameters.

Finally, the M-HSEG segmentation of the images was performed, using the three proposed implementations. The $L 1$ norm and the SAM between the region mean vectors were applied as DCs. ${ }^{2}$ In all experiments, the M-HSEG algorithm has been run until no more merging was possible. By assigning

\footnotetext{
${ }^{2}$ The $\infty$-norm was also applied as a DC; the corresponding results were not an improvement compared to the ones reported in this paper.
}

the class of each marker to the region containing this marker, the spectral-spatial classification maps were obtained.

Tables I and II summarize the global and class-specific accuracies of the pixelwise SVM classification and the proposed method, using Proba-MS and Morpho-MS marker selection approaches, respectively, and $S_{w g h t}=[0.0,0.2,0.5]$. The following measures of accuracy were applied: Overall Accuracy (OA is the percentage of correctly classified pixels), Average Accuracy (AA is the percentage of correctly classified pixels for each class) and kappa coefficient $\kappa$ [35]. In order to compare the results of the proposed method with other advanced classification techniques, we have included results obtained using the construction of an MFS from the same sets of markers (see Section IV). Fig. 5 shows some of the corresponding classification maps. We have also included in Table II accuracies of classification by majority voting within the neighborhoods defined by both watershed $(W H+M V)$ and HSEG segmentation $\left(H S E G+M V\right.$, with the parameter $S_{w g h t}=0.0$, which is equivalent to HSWO, and the SAM DC) [16]. The optimal hierarchical level for $H S E G+M V$ was chosen interactively and validated by quantitatively evaluating classification accuracies of the considered method at several hierarchical levels versus 


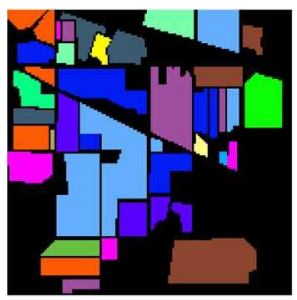

(a)

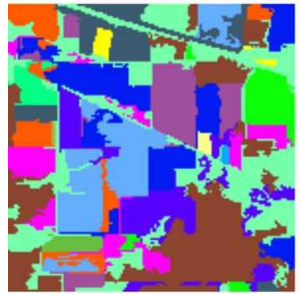

(d)
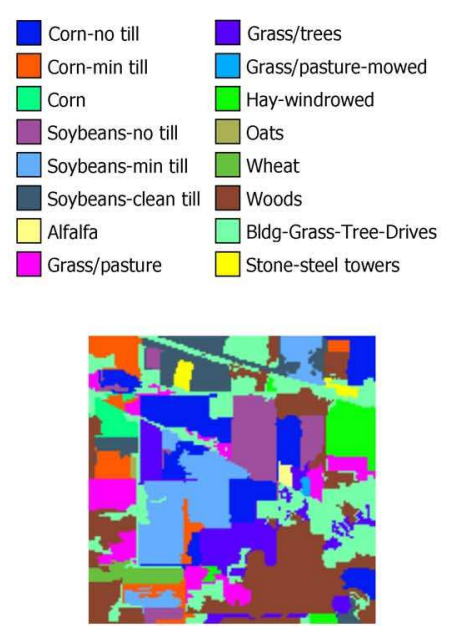

(e)

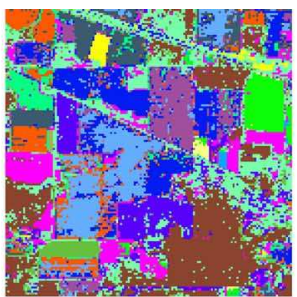

(b)

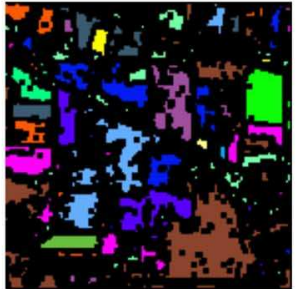

(f)

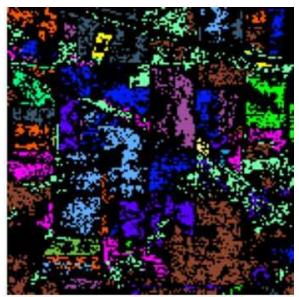

(c)

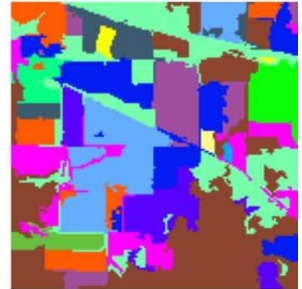

(g)

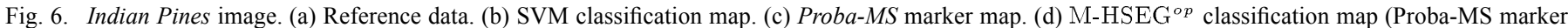

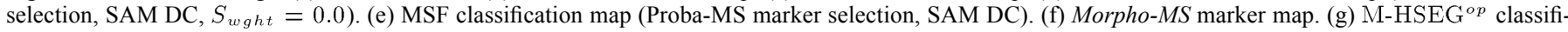
cation map (Morpho-MS marker selection, Inf DC, $S_{w g h t}=0.0$ ).

TABLE II

Global and Class-Specific ACCuracies in Percentage for the CENTER of PaVia IMAge, USIng the Morpho-MS MARKER SELECTION Approach AND USING THE PREVIOUSLY PROPOSED METHODS. The Highest AcCuracies are Bolded IN EACH CATEgory

\begin{tabular}{|c|c|c|c|c|c|c|c|}
\hline & \multicolumn{3}{|c|}{$\mathrm{M}-\mathrm{HSEG}^{o p}$} & \multicolumn{2}{|c|}{ MSF } & WH & $\overline{\text { HSEG }}$ \\
\hline$\overline{\mathrm{DC}}$ & L1 & SAM & SAM & L1 & SAM & + & + \\
\hline$\overline{S_{w g h t}}$ & & & 0.2 & - & - & MV & MV \\
\hline$\overline{\mathrm{OA}}$ & 97.95 & 98.00 & 97.89 & 97.70 & 98.13 & 97.14 & 97.15 \\
\hline AA & 97.66 & 97.75 & 97.66 & 97.37 & 98.13 & 96.41 & 96.41 \\
\hline$\kappa$ & 97.39 & 97.46 & 97.32 & 97.08 & 97.62 & 96.38 & 96.38 \\
\hline Water & 98.17 & 97.93 & 97.93 & 97.98 & 97.87 & 98.27 & 98.27 \\
\hline Trees & 95.41 & 94.27 & 94.27 & 94.19 & 94.32 & 91.62 & 91.66 \\
\hline Meadows & 95.79 & 96.30 & 96.30 & 96.19 & 97.33 & 93.97 & 93.97 \\
\hline Bricks & 93.03 & 94.88 & 94.74 & 89.86 & 96.02 & 96.82 & 96.82 \\
\hline Bare sc & 99.98 & 99.94 & 99.31 & 99.63 & 99.96 & 98.92 & 98.92 \\
\hline Asphalt & 98.21 & 98.96 & 98.88 & 99.02 & 98.96 & 95.64 & 95.64 \\
\hline Bitumen & 99.15 & 97.98 & 97.98 & 99.47 & 99.36 & 93.74 & 93.74 \\
\hline Tile & 99.17 & 99.45 & 99.54 & 100 & 100 & 98.80 & 98.80 \\
\hline Shadows & 100 & 100 & 100 & 100 & 99.40 & 99.90 & 99.90 \\
\hline
\end{tabular}

the test data. In our previous works, we have shown that the MSF-based and the majority voting-based methods used here for comparison yield better accuracies when compared to the well known ECHO technique [9], [16] and the approach based on SVM and Extended Morphological Profiles (EMP) [11], [15].

The following conclusions can be drawn:

- The proposed marker-based M-HSEG method yields accurate segmentation and classification results. The average accuracy is improved by 2.9 and 5.2 percentage points when compared to the SVM classification, when applying the Proba-MS and Morpho-MS marker selection approaches, respectively. Therefore, it is useful to include markers in the HSEG algorithm, in order to automatically select the relevant segmentation level.

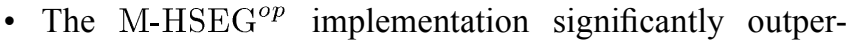
forms ${\mathrm{M}-\mathrm{HSEG}^{r}}^{r}$ and ${\mathrm{M}-\mathrm{HSEG}^{p}}^{p}$ implementations in terms of accuracies. Thus, a region mean vector seems to be a "poor" representative feature of image regions. It may be advantageous to apply more complex models for describing each region and dissimilarities between regions, for instance, statistical region model proposed in [36].

- The M-HSEG ${ }^{o p}$ method performs similar with $S_{w g h t}=$ 0.0 and $S_{w g h t}=0.2$ in terms of classification accuracies. However, classifications accuracies decrease with further increase of the $S_{w g h t}$ value.

- The Morpho-MS marker selection approach yields the highest classification accuracies for this data set.

- The proposed method using the Morpho-MS marker selection outperforms the $\mathrm{WH}+\mathrm{MV}$ and $\mathrm{HSEG}+\mathrm{MV}$ algorithms.

- The M-HSEG ${ }^{o p}$ classification results are similar to the MSF-based results. However, all the DCs give comparable accuracies when applying the $\mathrm{M}-\mathrm{HSEG}^{o p}$ approach. The MSF classification method using Proba-MS markers yields significantly lower accuracies when the SAM DC is applied. It assigns large portions of the water to the spatially adjacent asphalt regions, and assimilates shadows with neighboring regions (see Fig. 5(d)). Thus, the M-HSEG technique appears to be more robust when using different DCs.

\section{B. Classification of Indian Pines Image}

The Indian Pines image is of vegetation area that was recorded by the AVIRIS sensor in Northwestern Indiana. It is of 145 by 145 pixels, with a spatial resolution of $20 \mathrm{~m} /$ pixel. Twenty water absorption bands have been removed [37], and a 200-band image was used for our experiments. Sixteen information classes are considered, which are detailed in Fig. 6 and in Table III, with the number of samples for each class in the reference data. Fifty samples for each class were randomly selected from the reference data to be used as training samples, except for classes "alfalfa", "grass/pasture-mowed" and "oats". These classes contain a small number of samples in the reference data. Therefore, only 15 samples for each of these classes were randomly chosen as training samples. The remaining samples composed the test set. 
TABLE III

Results for the Indian Pines Data Set. Information Classes, Number of Labeled Samples, Global and Class-Specific Accuracies in Percentage, Using the Proba-MS Marker Selection Approach. The Highest Accuracies are Bolded in EACH CATEgORY

\begin{tabular}{|c|c|c|c|c|c|c|c|c|c|c|c|}
\hline \multirow{3}{*}{$\frac{\mathrm{DC}}{S_{w g}}$} & \multirow{3}{*}{$\begin{array}{l}\text { Number } \\
\text { of label. } \\
\text { samples }\end{array}$} & \multicolumn{2}{|c|}{$\mathrm{M}-\mathrm{HSEG}^{r}$} & \multicolumn{2}{|c|}{ M-HSEG $^{p}$} & \multicolumn{4}{|c|}{ M-HSEG ${ }^{o p}$} & \multirow{3}{*}{ SVM } & \multirow{2}{*}{$\begin{array}{l}\text { MSF } \\
\text { SAM }\end{array}$} \\
\hline & & SAM & Inf & SAM & Inf & SAM & Inf & SAM & Inf & & \\
\hline & & \multicolumn{6}{|c|}{0.0} & \multicolumn{2}{|c|}{0.2} & & \\
\hline Overall Accuracy & & 77.53 & 76.63 & 81.59 & 81.16 & 89.23 & 89.00 & 88.72 & 89.01 & 78.17 & 9.65 \\
\hline Average Accu & & 84.54 & 83.40 & 87.09 & 87.54 & 93.44 & 93.20 & 93.40 & 93.23 & 5.97 & 3.48 \\
\hline Карра С & & 74.48 & 73.40 & 79.10 & 78.62 & 87.72 & 87.47 & 87.15 & 87.49 & 5.33 & 3.19 \\
\hline Corn-no till & 1434 & 67.20 & 66.84 & 85.26 & 84.47 & 93.71 & 92.05 & 93.71 & 92.05 & 8.18 & 6.10 \\
\hline Corn-min till & 834 & 74.62 & 68.24 & 64.41 & 74.36 & 73.98 & 77.04 & 73.85 & 77.04 & 69.64 & 3.72 \\
\hline Corn & $2 ?$ & 64.67 & 59.78 & 64.67 & 67.39 & 96.74 & 95.11 & 96.74 & 95.65 & 91.85 & 5.65 \\
\hline till & & 71.57 & 71.79 & 83.66 & 75.49 & 99.02 & 99.02 & 99.02 & 99.02 & 2.03 & 8.04 \\
\hline & 24 & 3.16 & 71.59 & 68.90 & 67.12 & 75.68 & 75.02 & 74.52 & 75.02 & 95 & 7.01 \\
\hline So & 61 & 5.82 & 85.82 & 87.77 & 89.54 & 96.10 & 96.81 & 96.10 & 96.81 & 37.94 & 5.74 \\
\hline Alfalfa & 5 & 92.31 & 92.31 & 92.31 & 92.31 & 92.31 & 92.31 & 94.87 & 92.31 & 74.36 & 4.87 \\
\hline Gras & & 80.09 & 82.55 & 89.71 & 92.17 & 93.96 & 93.74 & 93.51 & 93.74 & 2.17 & 4.18 \\
\hline Grass & 7 & 77.19 & 72.02 & 83.07 & 86.23 & 97.85 & 97.42 & 97.85 & 97.42 & 91.68 & 97.85 \\
\hline & 2 & 100 & 10 & 100 & 100 & 100 & 100 & 100 & 100 & & 100 \\
\hline Hay-windrow & 48 & 99.54 & 99.54 & 98.41 & 99.54 & 99.54 & 99.32 & 99.54 & 99.32 & 97.72 & 99.54 \\
\hline Oats & 2 & 100 & 100 & 100 & 100 & 100 & 100 & 100 & 100 & 100 & 100 \\
\hline Wheat & 212 & 97.53 & 98.15 & 98.15 & 98.15 & 99.38 & 99.38 & 99.38 & 99.38 & 98.77 & 99.38 \\
\hline Woods & 1294 & 99.20 & 92.28 & 97.67 & 94.05 & 98.47 & 98.47 & 96.95 & 98.47 & 93.01 & 98.39 \\
\hline Bldg-G & 380 & 76.97 & 77.88 & 83.94 & 84.24 & 82.73 & 82.12 & 82.73 & 82.12 & 61.52 & 79.70 \\
\hline Stone-steel towers & 95 & 97.78 & 95.56 & 95.56 & 95.56 & 95.56 & 93.33 & 95.56 & 93.33 & 97.78 & 95.56 \\
\hline
\end{tabular}

A pixelwise classification on the 200-band image was performed using the multiclass one-versus-one SVM classifier with the Gaussian RBF kernel and parameters chosen by fivefold cross validation: $C=128$ and $\gamma=2^{-6}$. Then, marker selection using the two proposed approaches was performed, with the same parameters as for the Center of Pavia image (see Fig. 6). Finally, M-HSEG classification was applied, with the SAM and Infinity norm DCs, and executed until no more merging was possible.

Tables III and IV give the global and class-specific accuracies of the SVM classification and the proposed M-HSEG technique, using Proba-MS and Morpho-MS marker selection algorithms, respectively, and $S_{w g h t}=[0.0,0.2]$. The performances of the proposed approach are compared with those obtained by constructing an MSF from the same sets of markers, using the SAM DC. Table IV also contains results of the WH+MV and HSEG+MV techniques, applied in the same way as for the Center of Pavia image. Some of the corresponding classification maps are depicted in Fig. 6.

From the tables, similar conclusions as for the previous data set can be derived. The global and most of the class-specific accuracies are significantly improved when compared to the SVM classification. The M-HSEG ${ }^{o p}$ performs the best among the three proposed implementations. It gives similar results with $S_{w g h t}=0.0$ and $S_{w g h t}=0.2$. The average accuracy of the M-HSEG ${ }^{o p}$ classification is improved by 7.5 and 1.4 percentage points when compared to the SVM results, when applying Proba-MS and Morpho-MS marker selection algorithms, respectively.

The Morpho-MS marker selection approach yields higher overall, but lower average classification accuracies, when compared to the Proba-MS method. This is due to the fact that when applying the Morpho-MS technique, regions smaller than the size of the SE are not captured in a marker map, and thus they disappear in the final segmentation and classification maps. The corresponding classes to which these region belong ("oats" class) are not accurately classified. The Proba-MS
TABLE IV

GLOBAL AND Class-SPECIFIC ACCURACIES IN PERCENTAGE FOR THE INDIAN Pines IMAGe, USING THE MORPHO-MS MARKER SELECTION APPROACH AND Using the Previously Proposed Methods. The Highest Accuracies ARE BOLDED IN EACH CATEGORY

\begin{tabular}{|c|c|c|c|c|c|}
\hline \multirow[b]{2}{*}{$\overline{\mathrm{DC}}$} & \multicolumn{2}{|c|}{ M-HSEG ${ }^{o p}$} & MSF & \multirow{3}{*}{$\begin{array}{c}\text { WH } \\
+ \\
\text { MV }\end{array}$} & \multirow{2}{*}{$\begin{array}{c}\text { HSEG } \\
+\end{array}$} \\
\hline & SAM & Inf & SAM & & \\
\hline$S_{w g h t}$ & \multicolumn{2}{|c|}{0.0} & & & MV \\
\hline Overall Accuracy & 90.91 & 91.05 & 91.69 & 86.63 & 90.86 \\
\hline verage Accuracy & 7.10 & 87.36 & 7.99 & 1.61 & 3.9 \\
\hline Kappa coeff. $\kappa$ & 89.63 & 89.79 & 0.52 & 4.83 & 9.5 \\
\hline orn- & 90.97 & 91.55 & 91.84 & 94.22 & 90.46 \\
\hline & 91.58 & 91.33 & 92.60 & 8.06 & 3.0 \\
\hline & 8.91 & 95.65 & 5.65 & 8.59 & 95.65 \\
\hline & 97.93 & 96.51 & 7.0 & 6.30 & 92.0 \\
\hline & 82.13 & 81.39 & 2.34 & 8.82 & 4.04 \\
\hline & 96.63 & 96.28 & 6.28 & 90.78 & 95.3 \\
\hline & 92.31 & 92.31 & 94.87 & 94.87 & 92.3 \\
\hline & 92.17 & 92.17 & 94.63 & 95.08 & 94.41 \\
\hline & 6.84 & 97.56 & 97.85 & 97.99 & 97.5 \\
\hline & 100 & 100 & 100 & 100 & 100 \\
\hline Hay-w & 99.77 & 99.77 & 99.77 & 99.54 & 99.54 \\
\hline Oats & 0.00 & 0.00 & 0.00 & 100 & 100 \\
\hline & 99.38 & 98.15 & 100 & 99.38 & 98.15 \\
\hline & 99.28 & 99.60 & 10 & 97.11 & 98.63 \\
\hline & 57.88 & 70.00 & 69.39 & 69.39 & 82.12 \\
\hline Stone-steel towers & 97.78 & 95.56 & 95.56 & 95.56 & 100 \\
\hline
\end{tabular}

method proves to be more robust when selecting markers for regions of different sizes.

The MSF-based technique yields higher global accuracies when compared to the M-HSEG results for this data set, but the difference between average accuracies of both approaches is less than $1 \%$. The proposed M-HSEG ${ }^{o p}$ method yields significantly higher accuracies when compared to the $\mathrm{WH}+\mathrm{MV}$ algorithm. Its average accuracy is only $0.5 \%$ lower when compared to the HSEG+MV technique, which gives the highest average accuracy, but where an appropriate level of segmentation detail was chosen interactively. As a conclusion, classification results obtained with the proposed marker-based HSEG are comparable to the ones obtained by using either an interactive approach or the test data for selecting an optimal level of segmentation detail from the HSEG results. 
TABLE V

Results for the Hungarian Data Set. Information Classes, Number of Labeled Samples and Classification Accuracies in Percentage, Using the SAM Dissimilarity Criterion: Overall ACCuRACy (OA), Average ACCURACy (AA), Kappa Coefficient $(\kappa)$ AND Class-Specific Accuracies. The Highest Accuracies are Bolded IN Each Category

\begin{tabular}{|c|c|c|c|c|c|c|c|c|c|c|}
\hline \multirow{3}{*}{$\begin{array}{l}\text { Marker selection } \\
\text { Classification }\end{array}$} & \multirow{3}{*}{$\begin{array}{l}\text { Number } \\
\text { of labeled } \\
\text { samples }\end{array}$} & \multicolumn{3}{|c|}{ Proba-MS } & \multicolumn{3}{|c|}{ Morpho-MS } & \multirow{3}{*}{ SVM } & \multirow{3}{*}{$\begin{array}{c}\text { WH } \\
+ \\
\text { MV }\end{array}$} & \multirow{3}{*}{$\begin{array}{c}\text { HSEG } \\
+ \\
\text { MV }\end{array}$} \\
\hline & & \multicolumn{2}{|c|}{ M-HSEG ${ }^{o p}$} & \multirow{2}{*}{$\frac{\text { MSF }}{-}$} & \multicolumn{2}{|c|}{ M-HSEG $^{\circ p}$} & \multirow{2}{*}{ MSF } & & & \\
\hline & & 0.0 & 0.2 & & 0.0 & 0.2 & & & & \\
\hline$\overline{\mathrm{OA}}$ & & 79.86 & 79.94 & 80.40 & 85.09 & 84.72 & 84.90 & 77.36 & 81.32 & 85.7 \\
\hline $\mathrm{A}$ & & 4.20 & 84.25 & 4.81 & 9.36 & 88.51 & 8.60 & & 53 & 88.23 \\
\hline$\kappa$ & & 74.81 & 74.91 & 75.47 & 81.24 & 80.79 & 80.98 & 1 & 76.52 & 32.1 \\
\hline Arabl & 10141 & 67.77 & 67.87 & 68.43 & 73.51 & 72.99 & 73.35 & 60 & 88.84 & 4.92 \\
\hline low & 5602 & 92.42 & 92.48 & 92.73 & 96.18 & 95 & 5.93 & & 6.08 & 7.3 \\
\hline Greel & 4085 & 87.25 & 87.25 & 87.93 & 90 & 91 & 91.17 & & 6.08 & 90.2 \\
\hline Leg & & 3 & 87 & 3 & 41 & 88 & 86.79 & & 35 & 86.37 \\
\hline & & 29 & 83.29 & 83.58 & 89.53 & 89 & 88.67 & & 57 & 37.41 \\
\hline & 5 & 0.05 & 80.24 & 80.55 & 86.96 & 86.75 & 87.31 & 73 & 32.97 & 89.4 \\
\hline Forest & 771 & 91.21 & 91.21 & 93.14 & 97.32 & 95.68 & 97.02 & 83.01 & 84.74 & 91.8 \\
\hline
\end{tabular}

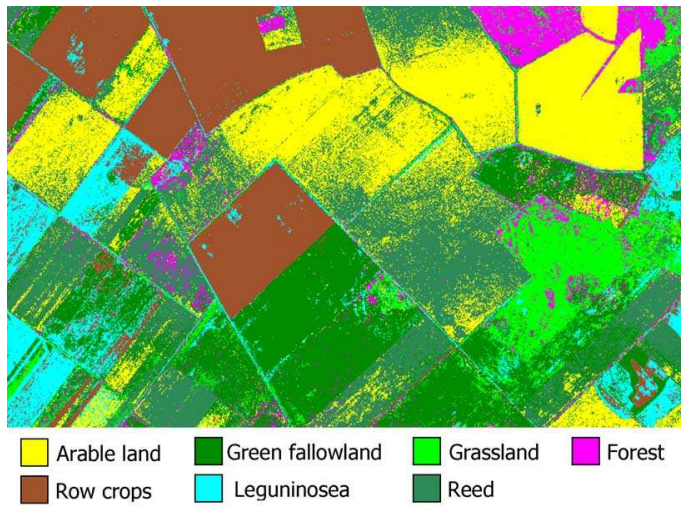

(a)

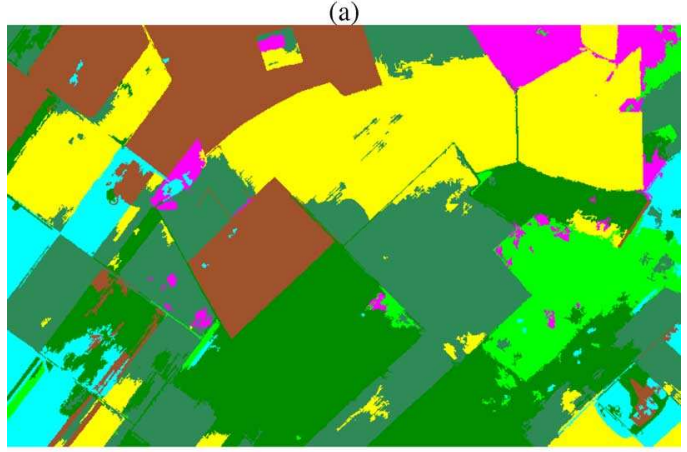

(b)

Fig. 7. Classification maps for the Hungarian image: (a) SVM map. (b) $\mathrm{M}-\mathrm{HSEG}^{\circ p}$ map, using Morpho-MS marker selection, SAM DC, $S_{w g h t}=0.0$.

\section{Classification of Hungarian Image}

The Hungarian hyperspectral image was recorded by the AISA Eagle sensor over the agricultural area of Heves, Hungary. The image is of 500 by 800 pixels, with a spatial resolution of $6 \mathrm{~m} /$ pixel. The number of spectral channels of the acquired image is 252 (with a spectral range from 395 to $975 \mathrm{~nm}$ ). The two most noisy channels were removed, and a 50-band image was used for experiments, obtained by averaging over every five adjacent bands [38]. The reference data contain seven classes of interest, detailed in Fig. 7 and in Table V, with the number of labeled pixels for each class. A hundred samples for each class were randomly chosen from the reference data as training samples, the remaining samples being used as the test set.
The one-versus-one SVM classification with the Gaussian RBF kernel was performed, with the parameters chosen by fivefold cross validation: $C=128$ and $\gamma=2^{-4}$ (see Fig. 7). Then, marker selection was applied in the same way as for the two previous data sets. Finally, both the M-HSEG and MSF classification were performed, using the SAM DC. Global and class-specific accuracies for the applied approaches, together with the $\mathrm{WH}+\mathrm{MV}$ and $\mathrm{HSEG}+\mathrm{MV}$ results, are given in Table V. ${ }^{3}$

The obtained results are consistent with those obtained for the two previous images. All spectral-spatial classification results are more accurate when compared to the pixelwise SVM result. The best average accuracy is achieved using the Morpho-MS marker selection followed by the M-HSEG ${ }^{o p}$ classification, with $S_{w g h t}=0.0$. It is improved by 9.3 percentage points when compared to the SVM classification. Fig. 7 shows the corresponding classification map, which contains more homogeneous regions when compared to the SVM result. The Hungarian image is composed of large regions mainly representing agricultural fields. Therefore, the Morpho-MS marker selection approach works very well for this data set, outperforming the Proba-MS technique in terms of accuracies. The M-HSEG ${ }^{o p}$ method with $S_{w g h t}=0.2$ performs better than with $S_{w g h t}=0.0$ for the Proba-MS set of markers in terms of accuracies, but worse for the Morpho-MS marker map.

As a conclusion, the experimental results on three data sets did show that the M-HSEG ${ }^{o p}$ with $S_{w g h t}=0.0$, i.e., best merge region growing controlled by classification-derived markers yields either the best or close to the best classification accuracies. Thus, it is recommended for spectral-spatial classification of hyperspectral images.

\section{CONCLUSIONS}

The HSEG segmentation approach is one of the few state-of-art segmentation algorithms that both naturally exploits spectral and spatial information and produces a hierarchical set of image segmentations. Many application areas can greatly benefit from methods able to automatically analyze segmentation hierarchies and select a single optimum segmentation level. One of such application areas is remotely sensed hyperspectral image classification, where segmentation can be used

\footnotetext{
${ }^{3}$ We have included in Table V only accuracies of the M-HSEG ${ }^{o p}$ implementation of the proposed approach, which are significantly higher compared to those obtained using $\mathrm{M}-\mathrm{HSEG}^{r}$ and $\mathrm{M}-\mathrm{HSEG}^{p}$ implementations.
} 
as a powerful tool for automatically defining both spatial and spectral dependencies in an image.

In this paper, a new marker-based Hierarchical SEGmentation (M-HSEG) method for the automated selection of a segmentation level from the segmentation hierarchy and hyperspectral image classification has been proposed. In this method, the automated choice of a single segmentation is achieved by using automatically selected markers. A marker map is first constructed using classification results. Two different classification approaches for automatic marker selection for M-HSEG are adapted and compared. Then, the novel M-HSEG algorithm is applied, resulting in a spectral-spatial classification map. Three different ways of integrating markers into the HSEG technique are proposed and investigated.

Experimental results, demonstrated on the three hyperspectral data sets, have shown that the new M-HSEG method yields accurate segmentation and classification maps and is sufficiently robust for classifying different kinds of images. Its performances are similar to the recently proposed MSF-based approach, which has been proven to outperform such standard spectral-spatial classification approaches as the ECHO and the SVM and EMP-based techniques. However, the proposed M-HSEG method has the following advantages when compared to the MSF-based technique:

- At each iteration, the dissimilarity criterion is computed between every two regions, while in the case of the MSF construction the dissimilarity criterion is computed between two pixels. Thus, if a relevant feature vector for every region is chosen, with the increase of the number of pixels in the region its feature vector can better represent an information class. This advantage is important for classifying images containing classes and spatial regions with high internal variance.

- M-HSEG provides a possibility of merging non-adjacent regions by clustering based on spectral information only. This may also lead to a more accurate and robust modeling and representation of classes of interest.

- M-HSEG produces a segmentation hierarchy. Thus, it remains possible to further analyze segmentation maps at multiple levels of detail, in order to select the most relevant segmentation map for a specific application.

It was concluded that a region mean vector feature may be not an accurate/sufficient representative of image regions. Therefore, in the future we plan to explore the choice of optimal representative features for segmentation regions, in particular texture and shape features, for further improving segmentation and classification results.

\section{ACKNOWLEDGMENT}

The authors would like to thank P. Gamba from the University of Pavia, Italy, D. Landgrebe from Purdue University, USA, BirdLife Hungary NGO and the University of Szent Istvan for providing the hyperspectral data.

\section{REFERENCES}

[1] H. Bagan, W. Takeuchi, T. Kinoshita, Y. Bao, and Y. Yamagata, "Land cover classification and change analysis in the Horqin sandy land from 1975 to 2007," IEEE J. Sel. Topics Appl. Earth Observ. Remote Sens., vol. 3, no. 2, pp. 168-177, Jun. 2010.
[2] C.-I. Chang, Hyperspectral Data Exploitation: Theory and Applications. New York: Wiley-Interscience, 2007.

[3] D. A. Landgrebe, Signal Theory Methods in Multispectral Remote Sensing. New York: Wiley, 2003.

[4] P. K. Goel, S. O. Prasher, R. M. Patel, J. A. Landry, R. B. Bonnell, and A. A. Viau, "Classification of hyperspectral data by decision trees and artificial neural networks to identify weed stress and nitrogen status of corn," Comput. Electron. Agricult., vol. 39, no. 2, pp. 67-93, 2003.

[5] C. H. Chen and P.-G. Peter Ho, "Statistical pattern recognition in remote sensing," Pattern Recognit., vol. 41, no. 9, pp. 2731-2741, 2008.

[6] J.-M. Yang, P.-T. Yu, and B.-C. Kuo, "A nonparametric feature extraction and its application to nearest neighbor classification for hyperspectral image data," IEEE Trans. Geosci. Remote Sens., vol. 48, no. 3, pp. 1279-1293, Mar. 2010.

[7] G. Camps-Valls and L. Bruzzone, "Kernel-based methods for hyperspectral image classification," IEEE Trans. Geosci. Remote Sens., vol. 43, no. 6, pp. 1351-1362, Jun. 2005.

[8] C. Chang and C. Lin, "LIBSVM - A Library for Support Vector Machines,” 2008 [Online]. Available: http://www.csie.ntu.edu.tw/ cjlin/ libsvm

[9] R. L. Kettig and D. A. Landgrebe, "Classification of multispectral image data by extraction and classification of homogeneous objects," IEEE Trans. Geosci. Electron., vol. 14, no. 1, pp. 19-26, Jan. 1976.

[10] X. Huang and L. Zhang, "A comparative study of spatial approaches for urban mapping using hyperspectral ROSIS images over Pavia city, northern Italy," Int. J. Remote Sens., vol. 30, no. 12, pp. 3205-3221, 2009.

[11] A. Plaza, J. A. Benediktsson, J. Boardman, J. Brazile, L. Bruzzone, G. Camps-Valls, J. Chanussot, M. Fauvel, P. Gamba, J. A. Gualtieri, M. Marconcini, J. C. Tilton, and G. Trianni, "Recent advances in techniques for hyperspectral image processing," Remote Sens. Environ., vol. 113, supplement 1, pp. S110-S122, 2009.

[12] Y. Tarabalka, J. Chanussot, and J. A. Benediktsson, "Segmentation and classification of hyperspectral images using minimum spanning forest grown from automatically selected markers," IEEE Trans. Syst., Man, Cybern.: B, vol. 40, no. 5, pp. 1267-1279, Oct. 2010.

[13] G. Camps-Valls, L. Gomez-Chova, J. Munoz-Mari, J. Vila-Frances, and J. Calpe-Maravilla, "Composite kernels for hyperspectral image classification," IEEE Geosci. Remote Sens. Lett., vol. 3, no. 1, pp. 93-97, Jan. 2006.

[14] M. Fauvel, J. Chanussot, J. A. Benediktsson, and J. R. Sveinsson, "Spectral and spatial classification of hyperspectral data using SVMs and morphological profiles," IEEE Trans. Geosci. Remote Sens., vol. 46, no. 11, pp. 3804-3814, Nov. 2008.

[15] Y. Tarabalka, J. Chanussot, and J. A. Benediktsson, "Segmentation and classification of hyperspectral images using watershed transformation," Pattern Recognit., vol. 43, no. 7, pp. 2367-2379, Jul. 2010.

[16] Y. Tarabalka, J. A. Benediktsson, J. Chanussot, and J. C. Tilton, "Multiple spectral-spatial classification approach for hyperspectral data," IEEE Trans. Geosci. Remote Sens., vol. 48, no. 11, pp. 4122-4132, Oct. 2010.

[17] R. Gonzalez and R. Woods, Digital Image Processing, 2nd ed. Englewood Cliffs, NJ: Prentice-Hall, 2002.

[18] K. Fu and J. Mui, "A survey on image segmentation," Pattern Recognit., vol. 13, no. 1, pp. 3-16, 1981.

[19] J.-M. Beaulieu and M. Goldberg, "Hierarchy in picture segmentation: A stepwise optimization approach," IEEE Trans. Pattern Anal. Machine Intell., vol. 11, no. 2, pp. 150-163, 1989.

[20] J. Tilton, "Image segmentation by region growing and spectral clustering with a natural convergence criterion," in Proc. IGARSS'08, 1998, vol. 4 , pp. 1766-1768.

[21] J. C. Tilton, "RHSEG User's Manual: Including HSWO, HSEG, HSEGExtract, HSEGReader and HSEGViewer," version 1.50, 2010, via email request to James.C.Tilton@nasa.gov.

[22] A. J. Plaza and J. C. Tilton, "Automated selection of results in hierarchical segmentations of remotely sensed hyperspectral images," in Proc. IGARSS'05, Jul. 2005, vol. 7, pp. 4946-4949.

[23] P. Soille, Morphological Image Analysis, 2nd ed. New York: Springer-Verlag, 2003.

[24] O. Gómez, J. A. González, and E. F. Morales, "Image segmentation using automatic seeded region growing and instance-based learning," in Proc. 12th Iberoamerican Congr. Pattern Recognit., Valparaiso, Chile, Nov. 2007, pp. 192-201.

[25] G. Noyel, J. Angulo, and D. Jeulin, "Morphological segmentation of hyperspectral images," Image Anal. Stereol., vol. 26, pp. 101-109, 2007. 
[26] G. Noyel, "Filtrage, Réduction de Dimension, Classification et Segmentation Morphologique Hyperspectrale," Ph.D. dissertation, Ctr. Mathematical Morphology, Paris Sch. Mines, Paris, France, 2008.

[27] L. Kaufman and P. J. Rousseeuw, Finding Groups in Data. An Introduction to Cluster Analysis. New York: Wiley, 1990.

[28] R. O. Duda, P. E. Hart, and D. G. Stork, Pattern Classification. New York: Wiley, 2001.

[29] G. Licciardi, F. Pacifici, D. Tuia, S. Prasad, T. West, F. Giacco, J. Inglada, E. Christophe, J. Chanussot, and P. Gamba, "Decision fusion for the classification of hyperspectral data: Outcome of the 2008 GRSS data fusion contest," IEEE Trans. Geosci. Remote Sens., vol. 47, no. 11, pp. 3857-3865, Nov. 2008.

[30] V. Vapnik, Statistical Learning Theory. New York: Wiley, 1998.

[31] L. Shapiro and G. Stockman, Computer Vision. Englewood Cliffs, NJ: Prentice-Hall, 2002.

[32] T.-F. Wu, C.-J. Lin, and R. C. Weng, "Probability estimates for multiclass classification by pairwise coupling," J. Machine Learning Res., no. 5, pp. 975-1005, 2004.

[33] J. Stawiaski, "Mathematical morphology and graphs: Application to interactive medical image segmentation," Ph.D. dissertation, Paris Sch. Mines, Paris, France, 2008

[34] R. C. Prim, "Shortest connection networks and some generalizations," Bell Syst. Tech. J., vol. 36, pp. 1389-1401, 1957.

[35] J. A. Richards and X. Jia, Remote Sensing Digital Image Analysis: An Introduction. New York: Springer-Verlag, 1999.

[36] S. Valero, P. Salembier, and J. Chanussot, "Comparison of merging orders and pruning strategies for binary partition tree in hyperspectral data," in Proc. ICIP'10, Hong Kong, 2010, pp. 1-4.

[37] S. Tadjudin and D. A. Landgrebe, "Covariance estimation with limited training samples," IEEE Trans. Geosci. Remote Sens., vol. 37, no. 4, pp. 2113-2118, Jul. 1999.

[38] Y. Tarabalka, J. A. Benediktsson, and J. Chanussot, "Spectral-spatial classification of hyperspectral imagery based on partitional clustering techniques," IEEE Trans. Geosci. Remote Sens., vol. 47, no. 8, pp. 2973-2987, Aug. 2009.

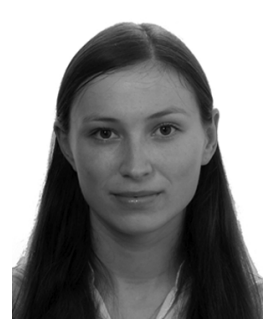

Yuliya Tarabalka (S'08-M'10) received the B.S. degree in computer science from Ternopil Ivan Pul'uj State Technical University, Ukraine, in 2005, the M.Sc. degree in signal and image processing from the Grenoble Institute of Technology (INPG), France, in 2007, the Ph.D. degree in signal and image processing from INPG, and the Ph.D. degree in electrical engineering from the University of Iceland in 2010.

From July 2007 to January 2008, she was a Researcher with the Norwegian Defence Research Establishment, Norway. She is currently a Postdoctoral research fellow at the NASA Goddard Space Flight Center, Greenbelt, MD. Her research interests are in the areas of image processing, pattern recognition, hyperspectral imaging and development of efficient algorithms.

Dr. Tarabalka is a member of the IEEE Geoscience and Remote Sensing Society.

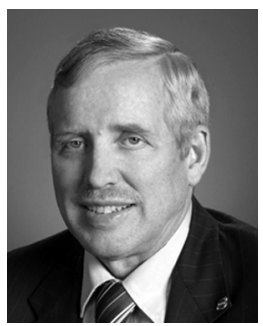

James C. Tilton (S'79-M'81-SM'94) received B.A. degrees in electronic engineering, environmental science and engineering, and anthropology and a M. E. E. (electrical engineering) from Rice University, Houston, TX in 1976. He also received an M. S. in optical sciences from the University of Arizona, Tucson, AZ in 1978 and a Ph.D. in electrical engineering from Purdue University, West Lafayette, IN in 1981.

$\mathrm{He}$ is currently a Computer Engineer with the Computational and Information Sciences and Technology Office (CISTO) of the Science and Exploration Directorate at the NASA Goddard Space Flight Center, Greenbelt, MD. As a member of the CISTO, Dr. Tilton is responsible for designing and developing computer software tools for space and earth science image analysis, and encouraging the use of these computer tools through interactions with space and earth scientists. His development of a recursive hierarchical segmentation algorithm has resulted in two patents and two other patent applications.

Dr. Tilton is a senior member of the IEEE Geoscience and Remote Sensing and Signal Processing Societies, and is a member of Phi Beta Kappa, Tau Beta Pi and Sigma Xi. From 1992 through 1996, he served as a member of the IEEE Geoscience and Remote Sensing Society Administrative Committee. Since 1996 he has served as an Associate Editor for the IEEE TRANSACTIONS ON Geoscience AND REMOte SENSING.

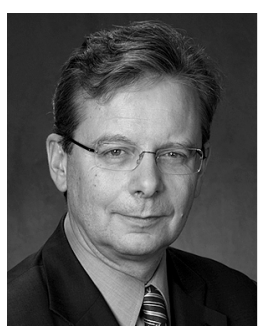

Jón Atli Benediktsson (S'84-M'90-SM'99-F'04) received the Cand.Sci. degree in electrical engineering from the University of Iceland, Reykjavik, in 1984, and the M.S.E.E. and Ph.D. degrees from Purdue University, West Lafayette, IN, in 1987 and 1990, respectively.

$\mathrm{He}$ is currently Pro Rector for Academic Affairs and Professor of Electrical and Computer Engineering at the University of Iceland. His research interests are in remote sensing, biomedical analysis of signals, pattern recognition, image processing, and signal processing, and he has published extensively in those fields.

Prof. Benediktsson is the President of the IEEE Geoscience and Remote Sensing Society for 2011-2012. He was Editor of the IEEE TRANSACTIONS ON GeOSCIENCE AND REMOTE SENSING (TGARS) from 2003 to 2008. He is a Fellow of the IEEE. He received the Stevan J. Kristof Award from Purdue University in 1991 as outstanding graduate student in remote sensing. In 1997, Dr. Benediktsson was the recipient of the Icelandic Research Council's Outstanding Young Researcher Award, in 2000, he was granted the IEEE Third Millennium Medal, in 2004, he was a co-recipient of the University of Iceland's Technology Innovation Award, in 2006 he received the yearly research award from the Engineering Research Institute of the University of Iceland, and in 2007, he received the Outstanding Service Award from the IEEE Geoscience and Remote Sensing Society. He is a member of Societas Scinetiarum Islandica and Tau Beta Pi.

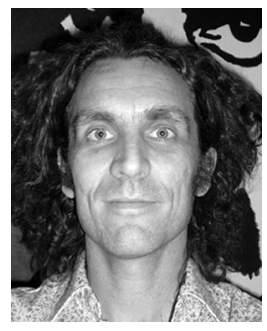

Jocelyn Chanussot (M'04-SM'04) received the M.Sc. degree in electrical engineering from the Grenoble Institute of Technology (Grenoble INP), Grenoble, France, in 1995, and the Ph.D. degree from Savoie University, Annecy, France, in 1998.

In 1999, he was with the Geography Imagery Perception Laboratory for the Delegation Generale de l'Armement (DGA - French National Defense Department). Since 1999, he has been with Grenoble INP, where he was an Assistant Professor from 1999 to 2005, an Associate Professor from 2005 to 2007, and is currently a Professor of signal and image processing. He is currently conducting his research at the Grenoble Images Speech Signals and Automatics Laboratory (GIPSA-Lab). His research interests include image analysis, multicomponent image processing, nonlinear filtering, and data fusion in remote sensing.

Dr. Chanussot is the founding President of IEEE Geoscience and Remote Sensing French chapter (2007-2010) which received the 2010 IEEE GRS-S Chapter Excellence Award "for excellence as a Geoscience and Remote Sensing Society chapter demonstrated by exemplary activities during 2009". $\mathrm{He}$ was a member of the IEEE Geoscience and Remote Sensing AdCom (2009-2010), in charge of membership development. He was the General Chair of the first IEEE GRSS Workshop on Hyperspectral Image and Signal Processing, Evolution in Remote Sensing (WHISPERS). He was the Chair (2009-2011) and Cochair (2005-2008) of the GRS Data Fusion Technical Committee. He was a member of the Machine Learning for Signal Processing Technical Committee of the IEEE Signal Processing Society (2006-2008) and the Program Chair of the IEEE International Workshop on Machine Learning for Signal Processing, (2009). He was an Associate Editor for the IEEE Geoscience and Remote Sensing Letters (2005-2007) and for Pattern Recognition (2006-2008). Since 2007, he is an Associate Editor for the IEEE Transactions on Geoscience and Remote Sensing. Since 2011, he is the Editor-in-Chief of the IEEE Journal OF SELECTED TOPICS IN APPLIED EARTH OBSERVATIONS AND REMOTE SENSING. 